\title{
Poly(hydrophobic amino acid) conjugates for the delivery of multiepitope vaccine against Group A Streptococcus
}

Armira Azuar ${ }^{1}$, Mohini A. Shibu ${ }^{1}$, Nomin Adilbish ${ }^{1}$, Nirmal Marasini ${ }^{2}$, Hong Hung ${ }^{1}$, Jieru Yang ${ }^{1}$, Yacheng Luo ${ }^{1}$, Zeinab G. Khalil ${ }^{3}$, Robert J. Capon ${ }^{3}$, Waleed M. Hussein ${ }^{1}$, Istvan Toth $^{1,3,4, *}$ and Mariusz Skwarczynski ${ }^{1, *}$

${ }^{1}$ School of Chemistry and Molecular Biosciences, The University of Queensland, St. Lucia, QLD 4072, Australia

2 School of Biomedical Sciences, The University of Queensland, St. Lucia, QLD 4072, Australia

${ }^{3}$ Institute of Molecular Bioscience, The University of Queensland, St. Lucia, QLD 4072, Australia

${ }^{4}$ School of Pharmacy, The University of Queensland, Woolloongabba, QLD 4102, Australia

*Corresponding author. Email: i.toth@uq.edu.au; Tel: (617) 33469892 (I.T.) and Email:

m.skwarczynski@uq.edu.au; Tel: (617) 33469894 (M.S.)

\section{Supporting Information}

Table S1 Vaccine dosage calculation (per mouse) for subcutaneous immunization

Figure S1 Analysis of the purified compounds 1-7 by analytical RP-HPLC and ESI-MS

Figure S2 HPLC monitoring of double conjugation reactions to produce multiepitope conjugate 7

Figure S3 Average opsonization percentage of different GAS strains 
Table S1. Vaccine dosage calculation (per mouse) for subcutaneous immunization

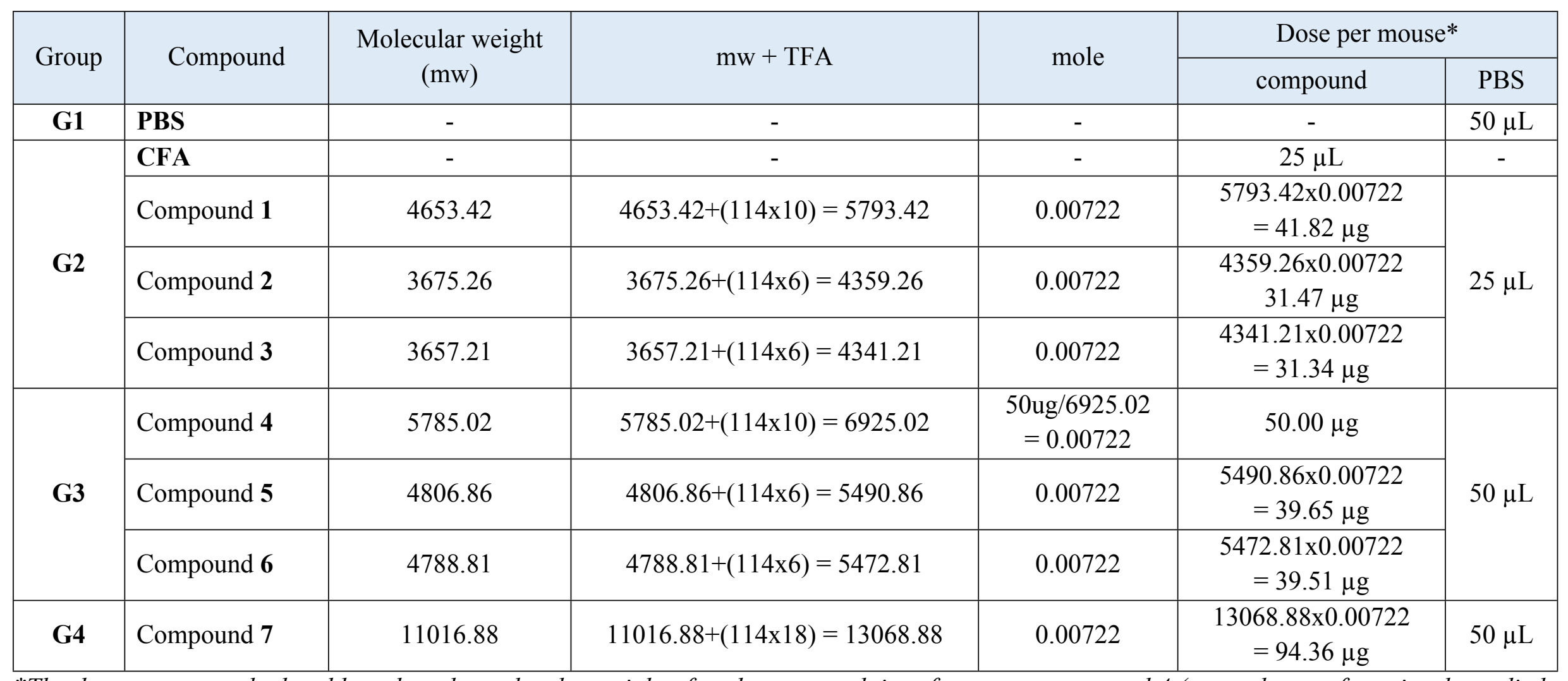

*The dosages were calculated based on the molecular weight of each compound, in reference to compound 4 (an analogue of previously studied compound) 


\section{Compound 1}
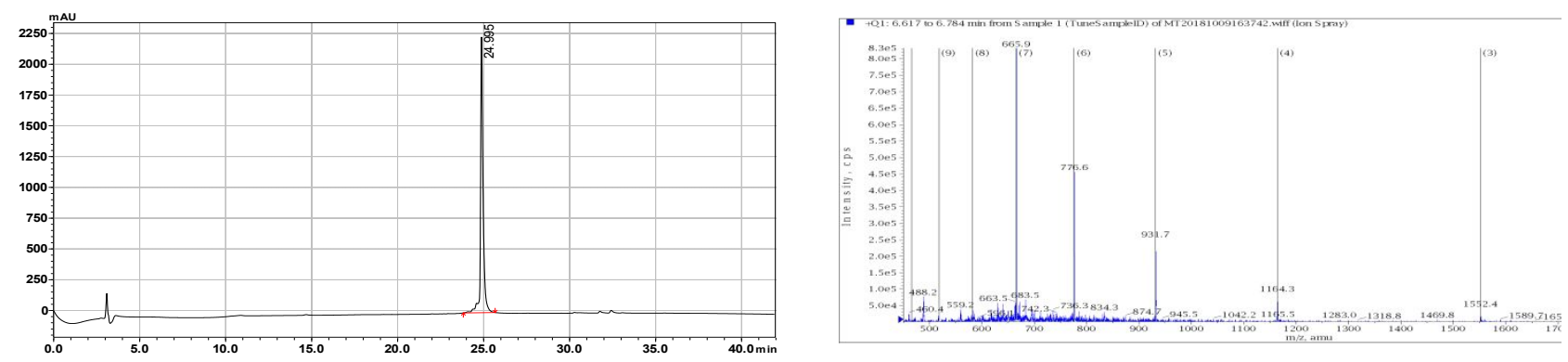

\section{Compound 2}
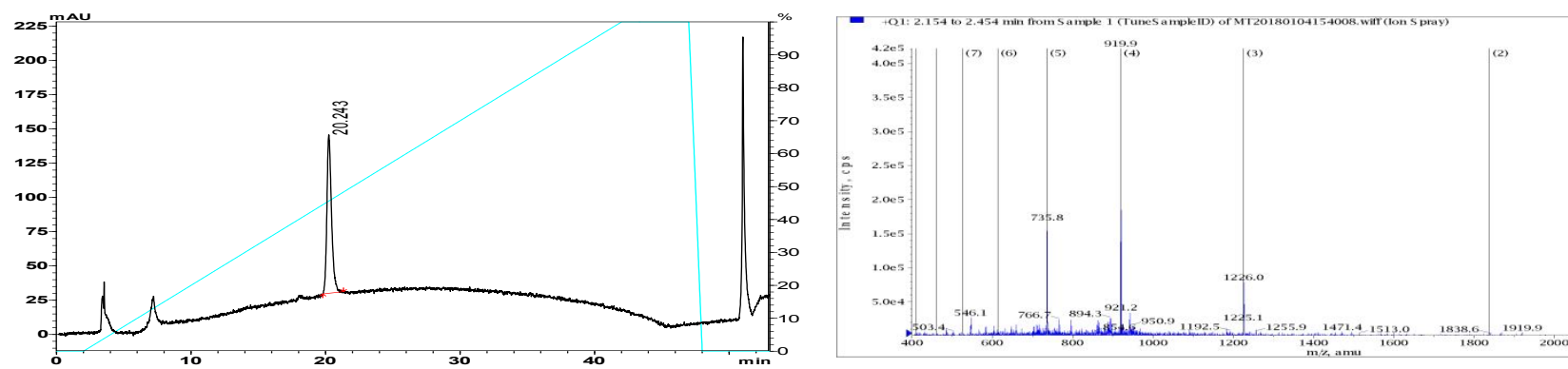

\section{Compound 3}
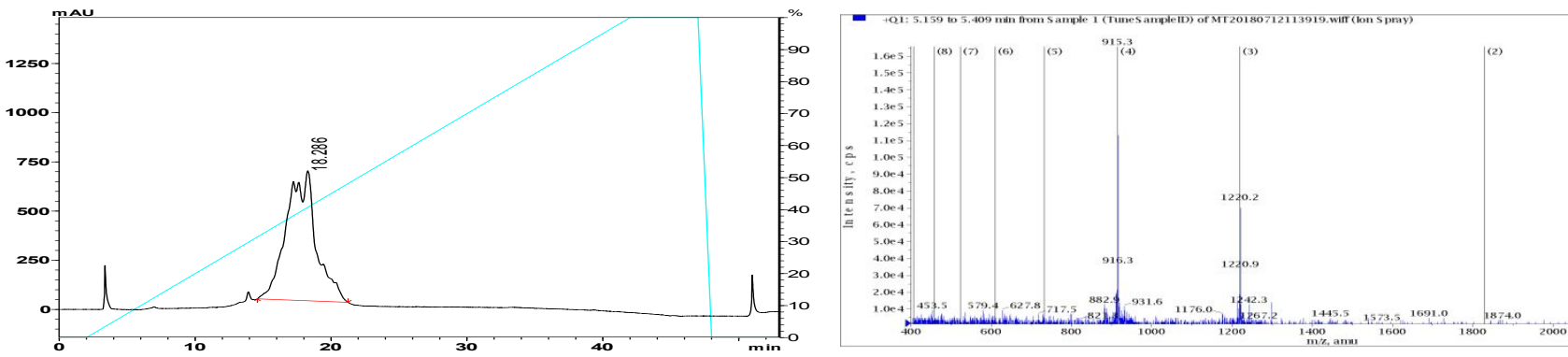

Compound 4
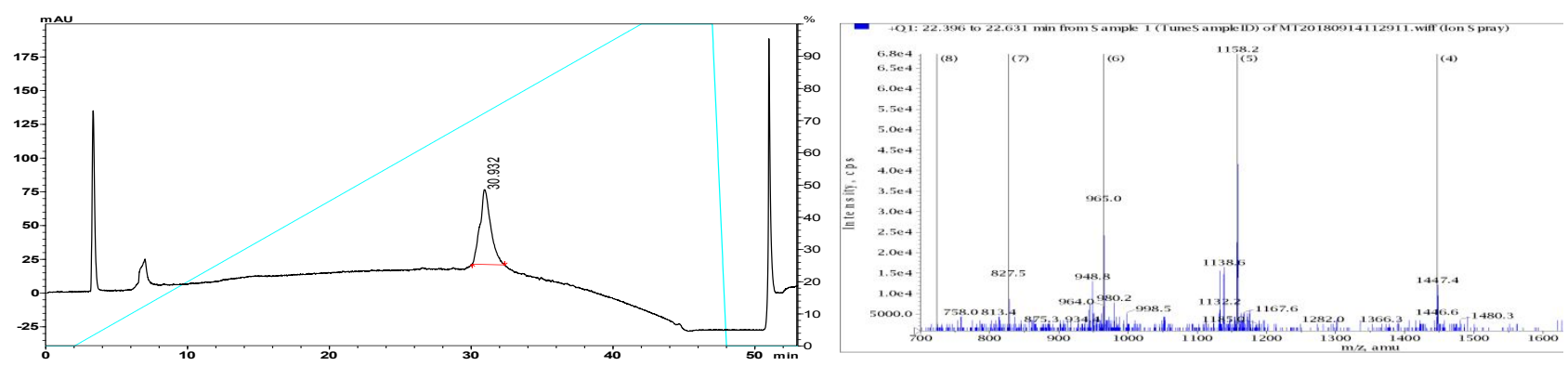

\section{Compound 5}
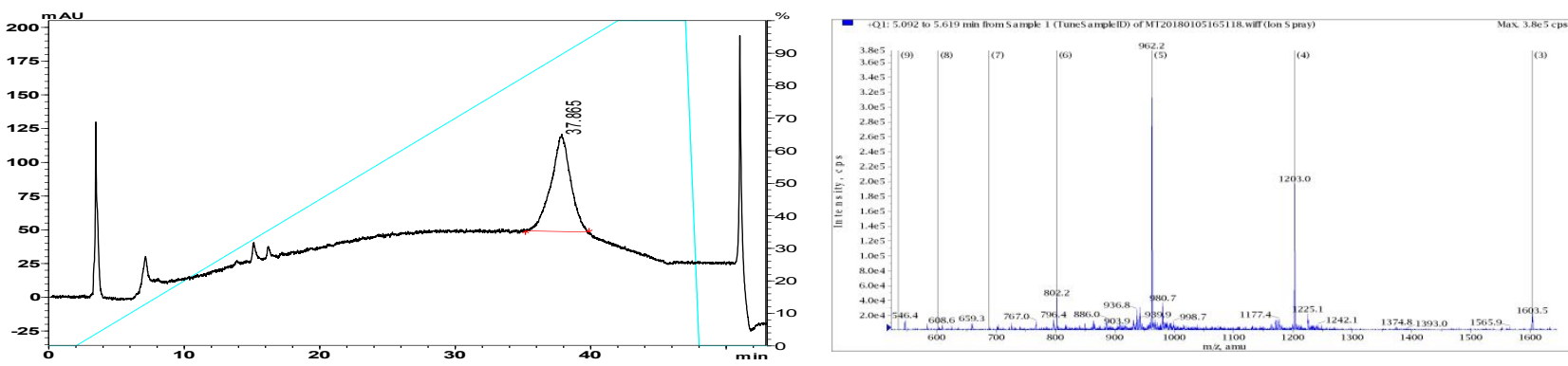
Compound 6
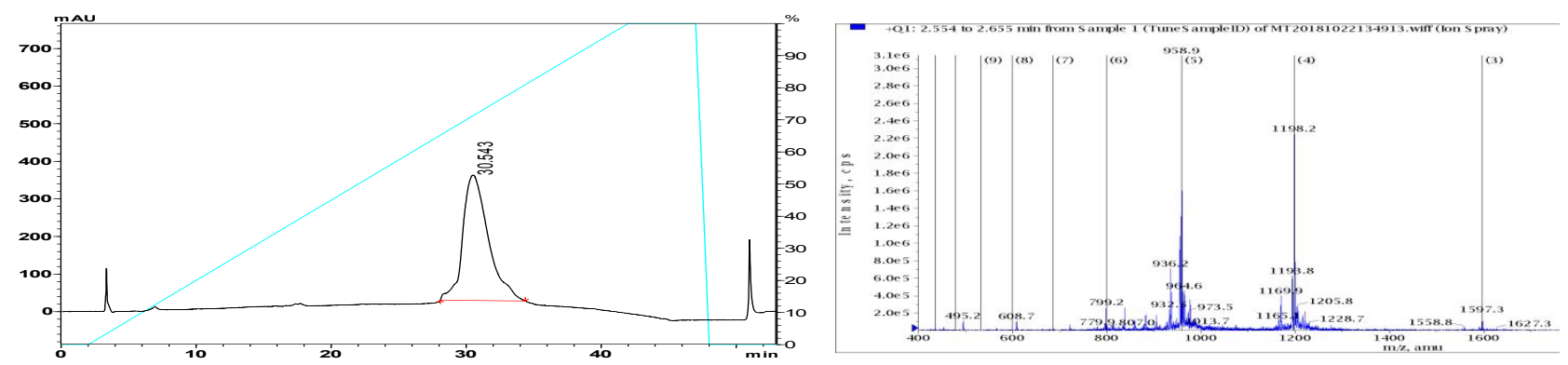

Compound $7 \mathbf{a}$
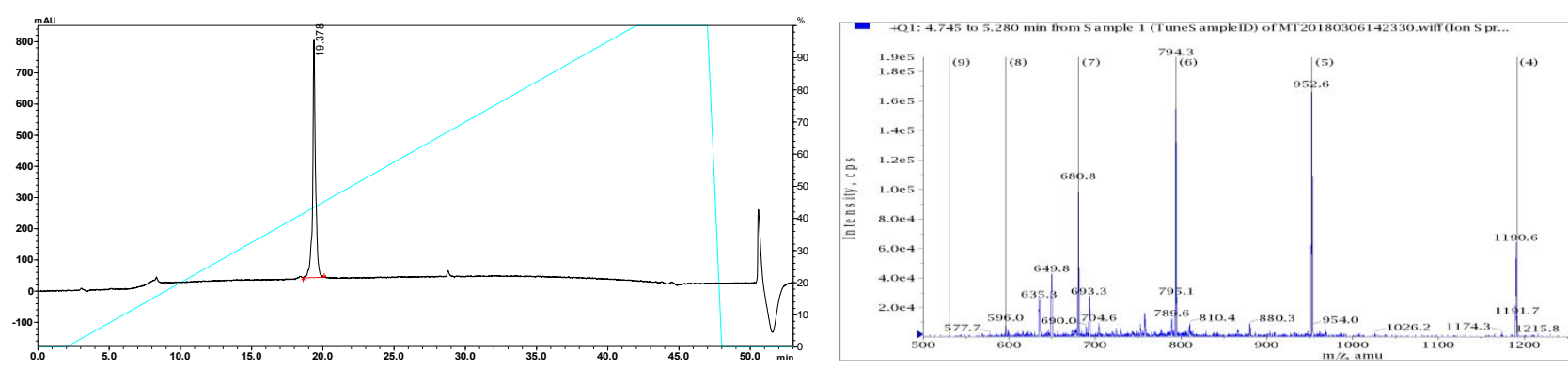

Compound Tb
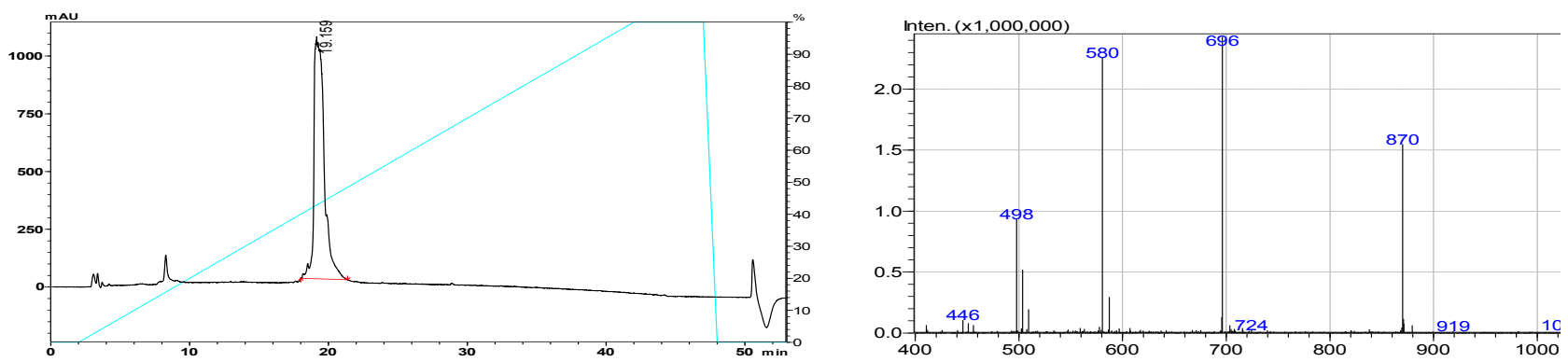

Compound Tc
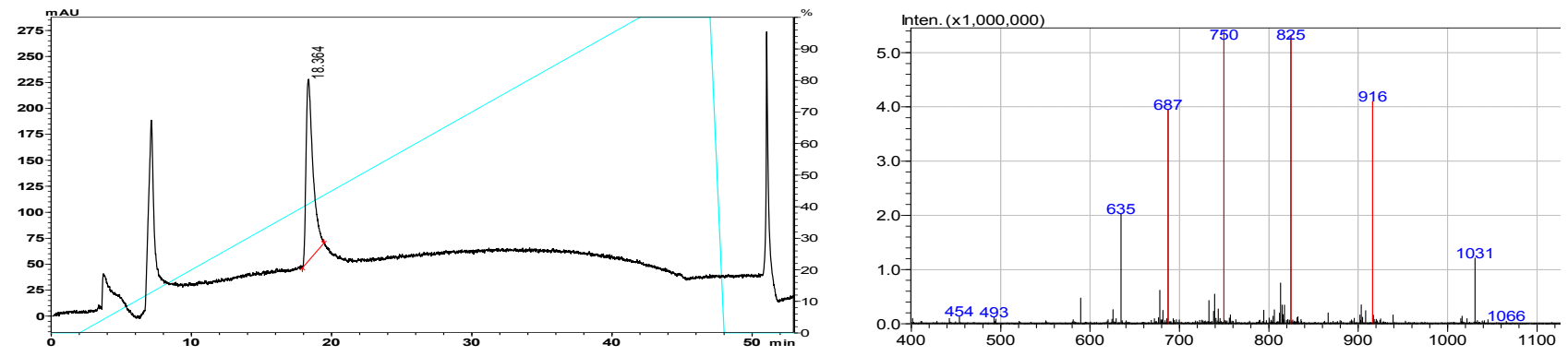

Compound Td
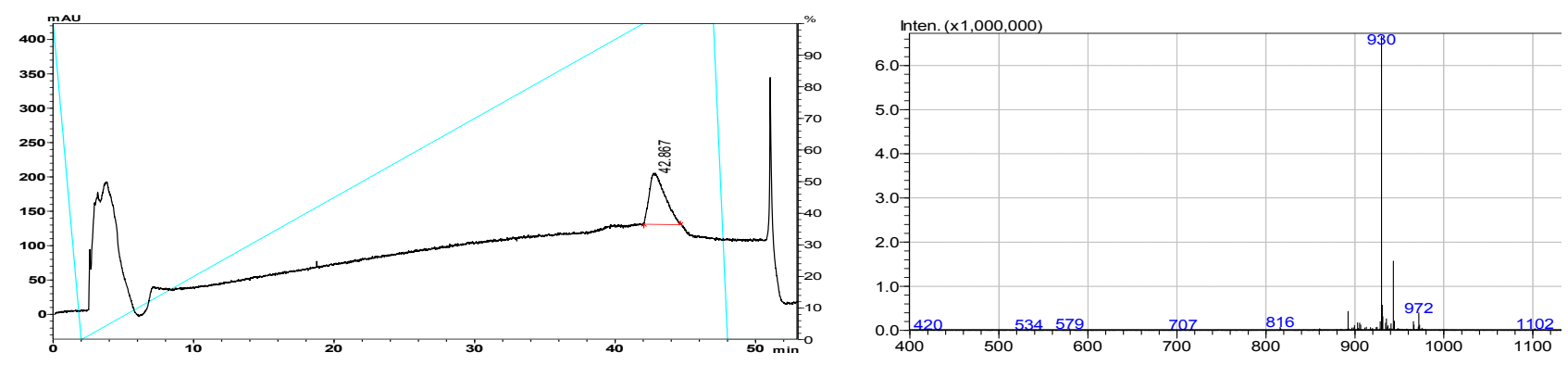


\section{Compound 7}
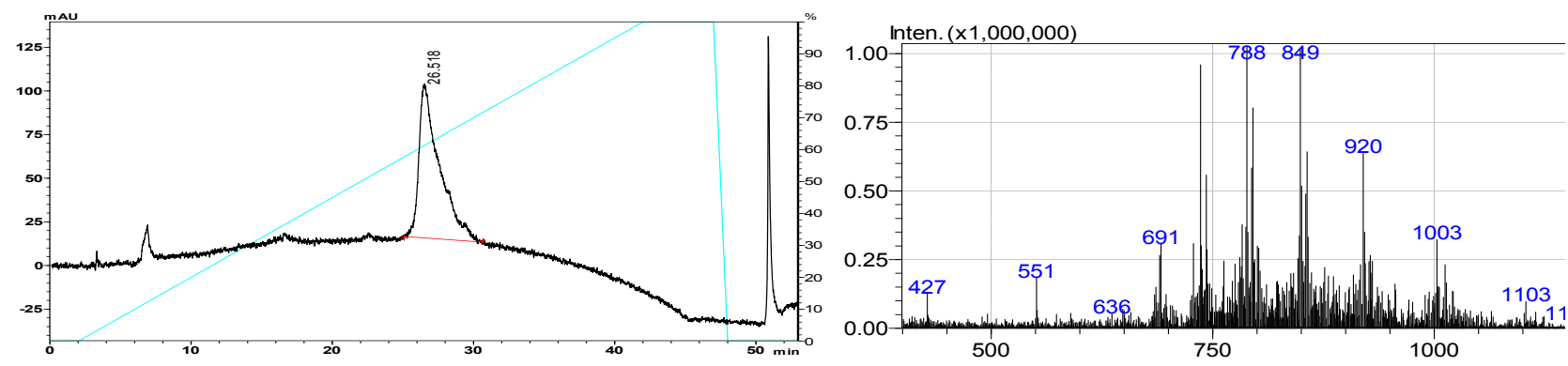

Figure S1. Analysis of the purified compounds 1-7 by analytical RP-HPLC and ESI-MS. The compounds were purified using preparative RP-HPLC with solvent B concentration gradient $25-45 \%$ (7a-7b), 30-50\% (1-3), 55-75\% (4-6), and 70-90\% (7d) from $\mathrm{R}_{\mathrm{t}} 5 \mathrm{~min}$ to $30 \mathrm{~min}$. and solvent B concentration gradient 25-65\% (7c) and 55-95\% (7) from $\mathrm{R}_{\mathrm{t}} 10 \mathrm{~min}$ to $40 \mathrm{~min}$. Analytical RP-HPLC graphs show pure compounds in single peak. The mass from these peaks, matched to the desired compounds in ESI-MS. 

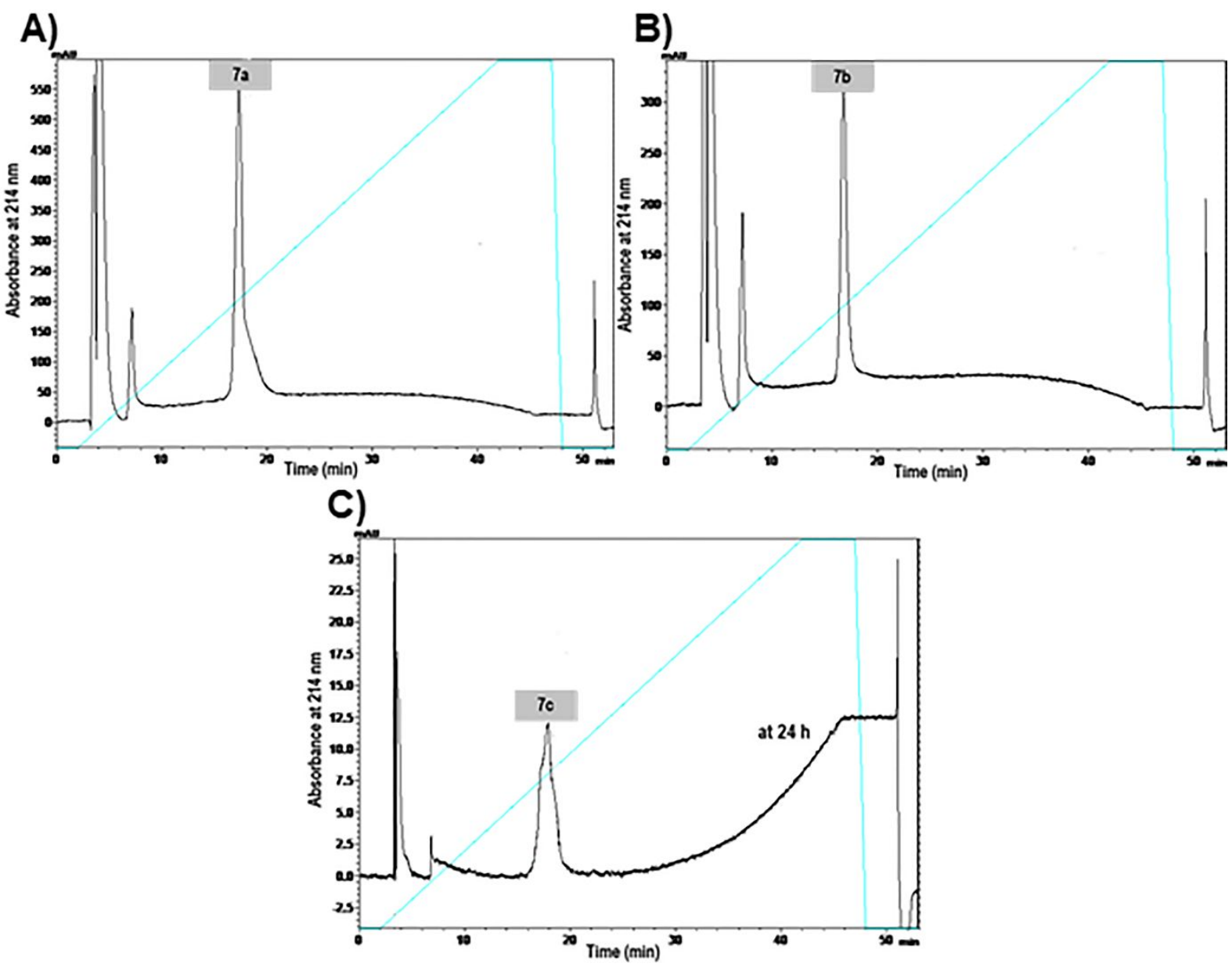

D)

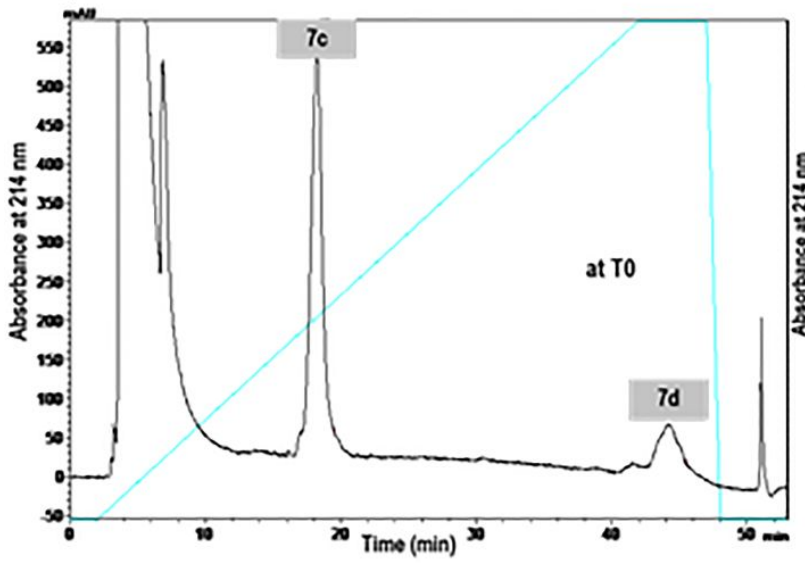

E)

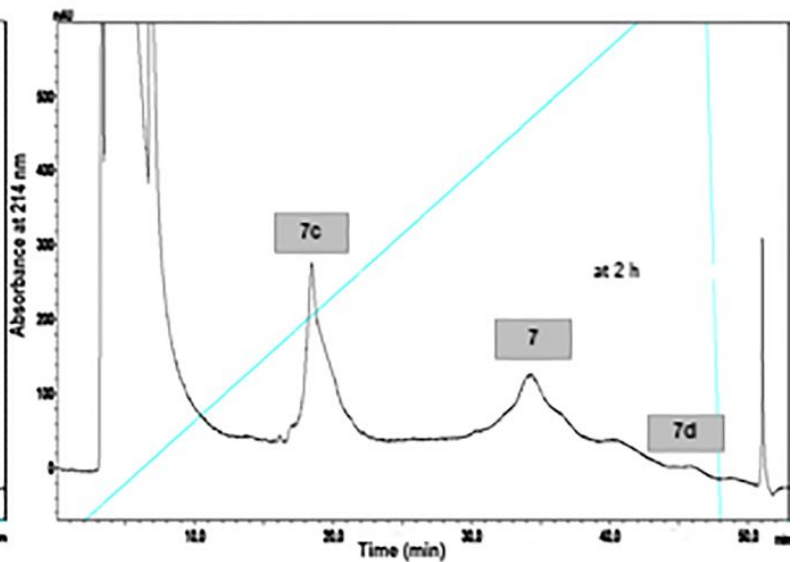

Figure S2. HPLC monitoring of two conjugation reactions to produce multiepitope conjugate 7. Mercapto-maleimide conjugation between a) azido-mercapto 7a used in excess and b) maleimide derivative $7 \mathbf{b}$ in guanidine buffer $\left(\mathrm{pH} 7.3\right.$ and $\left.37^{\circ} \mathrm{C}\right)$ to produce $\left.\mathbf{c}\right) 7 \mathbf{c}$ after 24 hours The second conjugation (CuAAC reaction) between conjugated product $7 \mathbf{c}$ and pHAA alkyne 7d was performed in DMF with presence of $\mathrm{Cu}$ wire at $50{ }^{\circ} \mathrm{C}$ under a nitrogen atmosphere d) at 0 time and after e) $2 \mathrm{~h}$. Solvent gradient ( $0 \%$ of solvent B to $100 \%$ for $40 \mathrm{~min}$ ) with compound detection at $214 \mathrm{~nm}$ was showed by blue line. 


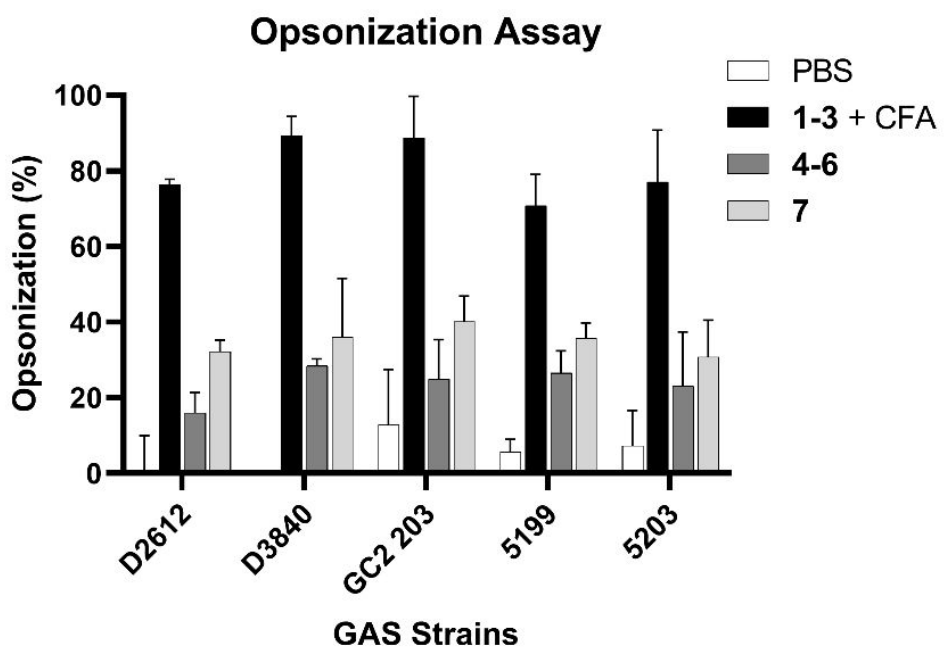

Figure S3. Average opsonization percentage of different GAS strains (D2612, D3840, GC2 203, ACM-5199, and ACM-5203) by serum collected on day 49 (after third boost). 